\title{
sols grenus sous fortes contraintes
}

\author{
par \\ M.P. Luong \\ et \\ A. Touati \\ Laboratoire de Mécanique des Solides \\ (E.P., E.N.P.C., E.N.S.M.) associé au C.N.R.S.
}

\section{Introduction}

Bien que de nombreuses études expérimentales ([5], [6], [7], [8], [9], [10], [16] et [17]) aient été effectuées sur le comportement mécanique des matériaux pulvérulents très divers, comme les sables, les graves et les enrochements, soumis à des fortes contraintes de confinement, les lois de comportement actuellement utilisées sont surtout construites sur la base des phénomènes se produisant dans les matériaux granulaires sous faibles contraintes, généralement inférieures à $1 \mathrm{MPa}$.

II faut donc se demander si l'extrapolation de tels modèles de comportement est justifiée dans l'analyse des problèmes géotechniques de plus en plus fréquents nécessitant une bonne compréhension du comportement mécanique des sols grenus sous fortes contraintes jusqu'à $50 \mathrm{MPa}$ : battage des pieux, compactage dynamique, fondations profondes, explosions, chocs, puits et tunnels à grande profondeur, barrages de grande hauteur, etc.

\section{Approche proposée}

Les sols pulvérulents sont des milieux formés de grains solides sans liaison effective les uns avec les autres. En première approximation, le comportement rhéologique d'un tel milieu résulte essentiellement des propriétés des grains élémentaires. Une telle approche a donné lieu à de nombreux travaux, allant de l'étude des contacts intergranulaires ([3], [4], [14]) jusqu'à la modélisation de l'arrangement de ces grains pour former la structure globale ([1], [2], [13]).

Pour représenter les milieux granulaires réels, on utilise des milieux idéalisés constitués d'assemblages réguliers de sphères égales ou d'arrangements de cylindres de différents diamètres. Ces considérations ont permis de saisir quelques aspects des mécanismes gouvernant les déformations du matériau. Cependant, elles s'avèrent souvent insuffisantes pour obtenir de bonnes corrélations avec le comportement réel observé.

On se propose d'étudier ici le comportement rhéologique des sols sableux soumis aux chargements monotone, statique et cyclique - cas où les forces d'inertie sont relativement négligeables - à l'aide de l'appareil triaxial axisymétrique conventionnel avec les notations suivantes (fig. 1):

- Contrainte moyenne $\mathrm{p}=\left(\sigma_{1}+2 \sigma_{3}\right) / 3$

- Contrainte déviatorique $\mathrm{q}=\sigma_{1}-\sigma_{3}$

- Niveau déviatorique $\eta=q / p$

auxquelles on associe les déformations

- volumique $\varepsilon_{v}=\varepsilon_{1}+2 \varepsilon_{3}$

- de distorsion $\varepsilon_{\mathrm{q}}=2\left(\varepsilon_{1}-\varepsilon_{3}\right) / 3$

- et le taux de dilatance $\delta=\dot{\varepsilon}_{\mathrm{v}} / \dot{\varepsilon}_{\mathrm{q}}$.

Les déformations réversibles et irréversibles sont respectivement notées $\varepsilon^{\theta}$ et $\varepsilon^{p}$.

\section{Matériaux étudiés}

Le choix s'est porté sur trois sables de dureté et de granulométrie différentes (figure 2):

- Sable de Fontainebleau ayant une granulométrie uniforme $0,08<\mathrm{d}<0,4 \mathrm{~mm}$, le coefficient de Hazen $d_{60} / d_{10}=1,48, d_{50}=0,17 \mathrm{~mm}$ et constitué de grains blancs siliceux assez anguleux;

- Sable de Loire à granulométrie étalée $0,08<\mathrm{d}<$ $2 \mathrm{~mm}$ de coefficient d'uniformité $\mathrm{d}_{60} / \mathrm{d}_{10}=1,95, \mathrm{~d}_{50}=$ $0,7 \mathrm{~mm}$ et contenant un faible pourcentage de grains siliceux;

- Sable de Seine à granulométrie plus étalée $0,08 \mathrm{~mm}<\mathrm{d}<5 \mathrm{~mm}, \mathrm{~d}_{60} / \mathrm{d}_{10}=3,2, \mathrm{~d}_{50}=0,63 \mathrm{~mm}$ et composé de grains siliceux avec un faible pourcentage de particules argileuses de couleur jaune, mélangées de calcaire.

Les caractéristiques de compacité sont données dans le tableau ci-dessous. 


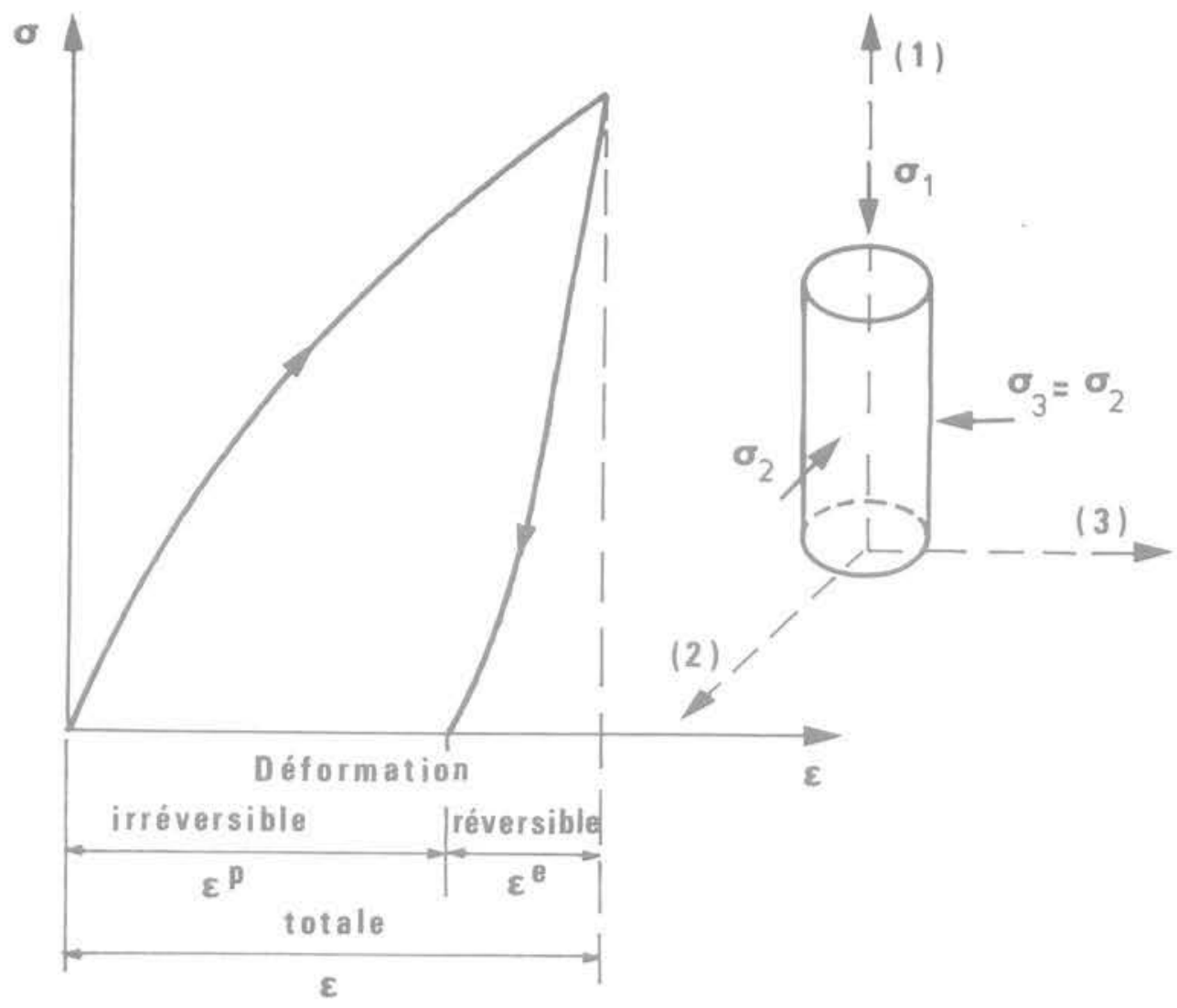

Fig. 1 Paramètres de l'essai triaxial asymétrique

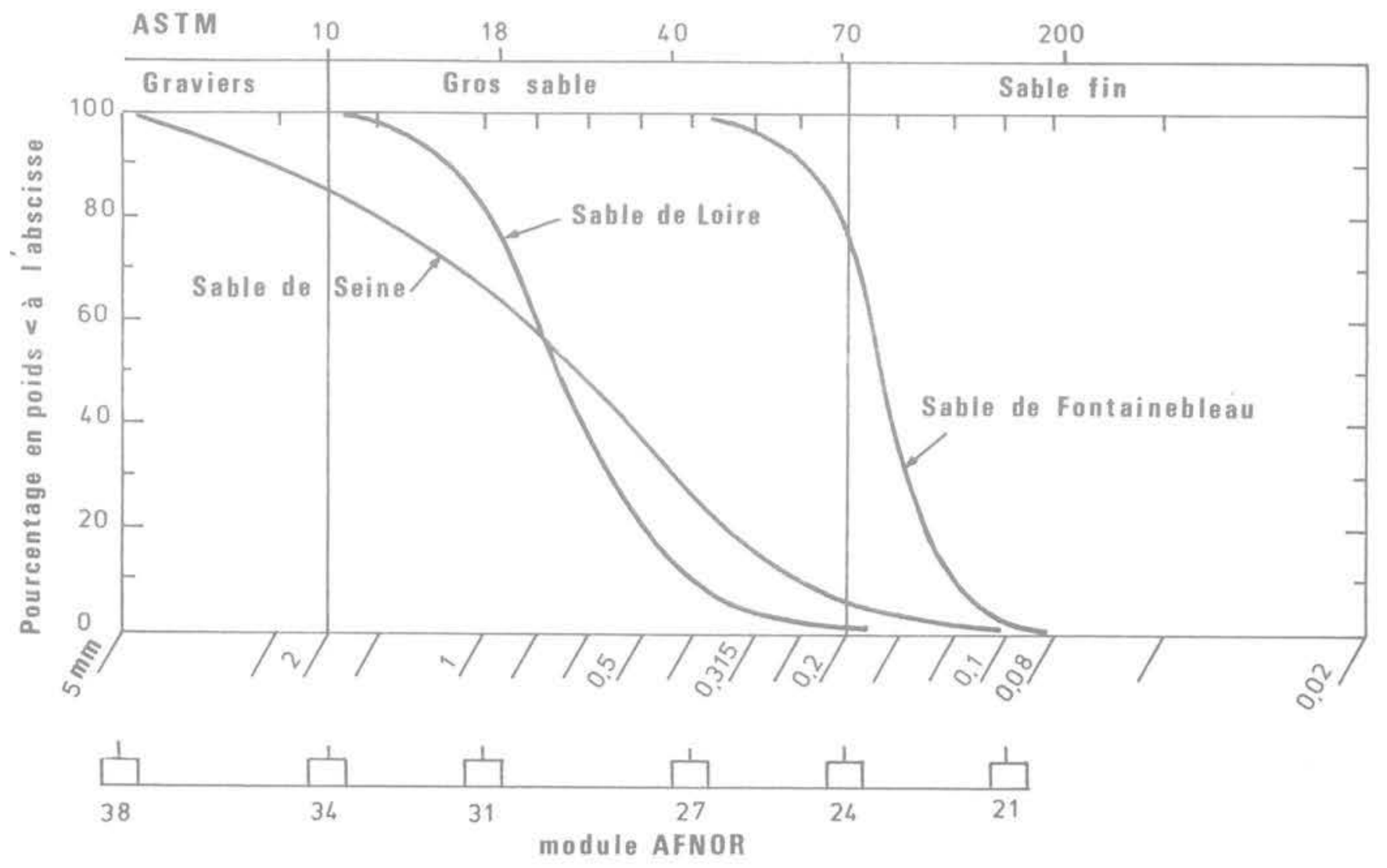

Fig. 2 Granulométries des trois sables étudiés 


\begin{tabular}{l|c|c|c|c|c}
\hline & $\gamma_{\mathrm{s}} \mathrm{kN} / \mathrm{m}^{3}$ & $\gamma_{\mathrm{d} \min } \mathrm{kN} / \mathrm{m}^{3}$ & $\gamma_{\mathrm{d} \max } \mathrm{kN} / \mathrm{m}^{3}$ & $e_{\max }$ & $e_{\min }$ \\
\cline { 2 - 6 } Sable de Fontainebleau & 26,36 & 13,6 & 17,1 & 0,940 & 0,540 \\
Sable de Loire & 27,29 & 13,9 & 17,2 & 0,963 & 0,587 \\
Sable de Seine & 26,69 & 14,9 & 18,6 & 0,790 & 0,435 \\
\hline
\end{tabular}

\section{Procédure expérimentale}

Les essais ont été effectuées en compression triaxiale (raccourcissement axial) et en extension triaxiale (rallongement axial) à vitesse de charge $\dot{\sigma}_{1}$ ou $\dot{\sigma}_{3}$ imposée en condition drainée ou non drainée suivant différents trajets de charge :

- trajet triaxial conventionnel à confinement constant;

- trajet à contrainte moyenne constante;

- trajet de chargement radial à niveau déviatorique constant;

- trajet de chargement isotrope.

Le chargement monotone - où l'échantillon est soumis à une vitesse de charge constante jusqu'à l'écoulement plastique - permet de suivre l'évolution des mécanismes de déformation du matériau avec le niveau de contrainte ainsi que l'influence du trajet de charge sur ces mécanismes.

Le chargement cyclique - où l'échantillon de sable est soumis à un ou plusieurs niveaux de contrainte moyenne et de contrainte déviatorique avec un faible nombre de cycles quasi-statiques (20 cycles au maximum) pour chaque niveau - nous renseigne sur le caractère pseudo-réversible du matériau et permet la détermination des paramètres physiques capables de décrire son comportement dans une modélisation numérique (paramètres élastiques).

Les chargements cycliques prolongés sur un grand nombre de cycles à amplitude constante décrit l'évolution du comportement du matériau en fonction de I'histoire du chargement et en particulier des déformations permanentes.

L'étalonnage et la précision relative des mesures de déformations volumiques sont précisées par la figure 3 qui montre l'évolution de la variation de volume $\left(\Delta \mathrm{V} / \mathrm{S}_{\mathrm{m}}\right)$ due à la pénétration d'une membrane d'épaisseur $1.5 \mathrm{~mm}$, qui devient asymptotiquement constante avec la pression de confinement $\sigma_{c}$; pour le sable de Fontainebleau de densité sèche 1,69-1,64, elle se stabilise à partir de $\sigma_{c}=5 \mathrm{MPa}$.

\section{Comportement sous contrainte isotrope}

Sous chargement isotrope, les caractéristiques contraintes-déformations d'un matériau granulaire dépendent de la densité initiale et de la contrainte moyenne: plus le matériau est lâche, plus la variation de volume due au mécanisme de serrage est grande et plus cette variation contient une grande partie irréversible lors du premier chargement.

Une série d'essais en compression isotrope sur le sable de Fontainebleau a été effectuée pour examiner les différents facteurs suivants :

- Amplitude des déformations irréversibles;

- Boucles d'hystérésis en fonction du niveau de la charge;

- Évolution de l'anisotropie initiale du matériau;

- Attrition des grains due à l'application d'un chargement monotone et cyclique.
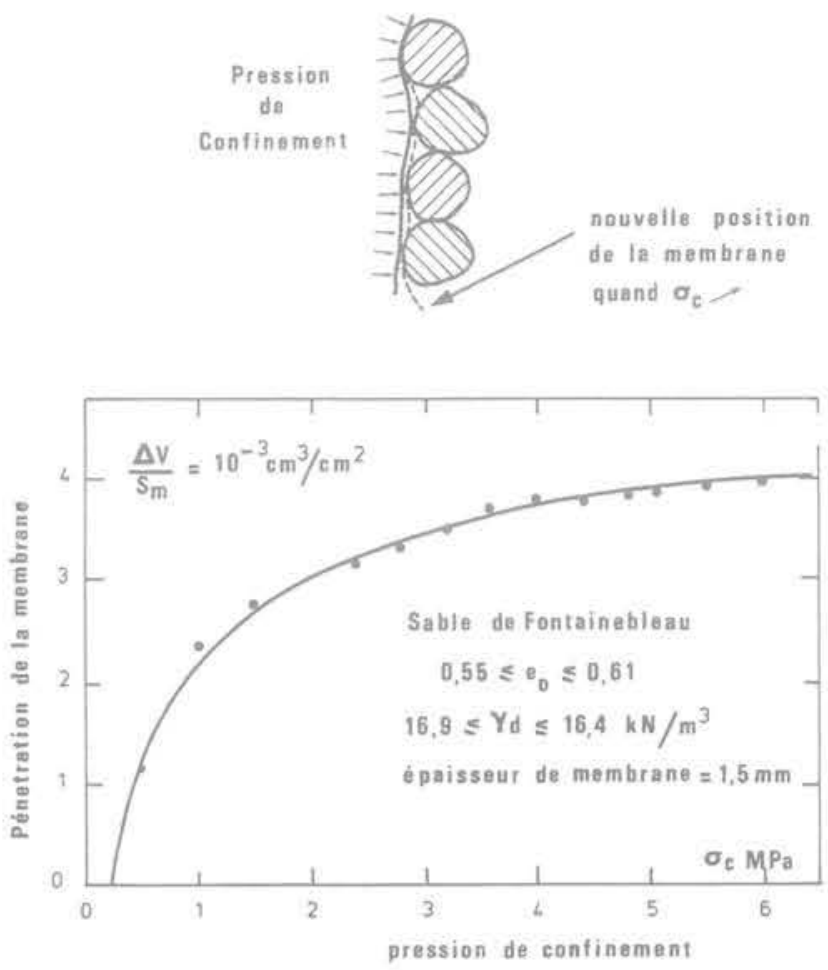

Fig. 3 Effet de pénétration de la membrane sur la mesure de variation de volume

\subsection{Chargement monotone}

La figure 4 montre, avec la même échelle pour les déformations volumiques totales, les courbes $\left(\Delta V / V_{0}, p\right)$ obtenues sur des échantillons mis en place à quatre densités différentes $\left(\gamma_{a}=15,34 ; 15,53 ; 16,34\right.$ et $16,91 \mathrm{kN} / \mathrm{m}^{3}$ ) et soumis à un cycle de charge-décharge isotrope de $p=6 \mathrm{MPa}$. On y constate clairement l'influence de la densité initiale sur la courbe effort-déformation. II est difficile de faire apparaître une courbe unique de consolidation isotrope du matériau, même pour des densités initiales pratiquement équivalentes. La décharge effectuée sur ce trajet de charge met en évidence l'importance croissante de la déformation irréversible cumulée au cours du premier chargement avec la porosité initiale du matériau.

La figure 5 représente pour un sable dense $\left(\gamma_{d}=\right.$ $16,46 \mathrm{kN} / \mathrm{m}^{3}$ ) la variation de volume irréversible $\left(\Delta \mathrm{V}^{\mathrm{P}} / \mathrm{V}_{\mathrm{o}}\right)$ par rapport au volume initial en fonction de la contrainte isotrope appliquée p.

La compressibilité irréversible apparaît très importante pour des valeurs faibles de la pression isotrope $(p<10 \mathrm{MPa})$. La concavité de la courbe effortdéformation est dirigée dans le sens des efforts croissants. Cette propriété traduit une diminution de la compressibilité relative avec l'augmentation de la pression. 


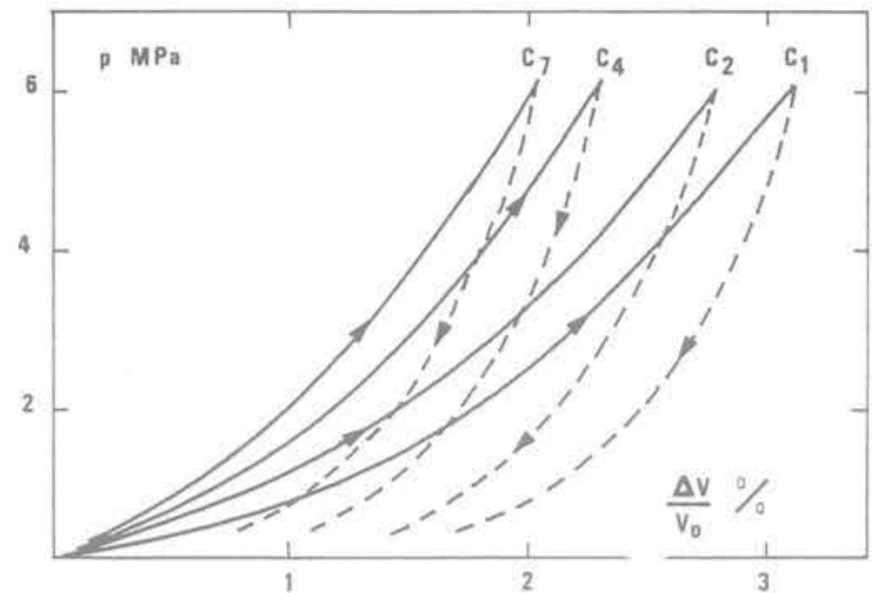

Sable de Fontainableau

$\begin{array}{cccc}c_{1} & a=0,717 & D r=55,6 \% & Y_{d}=15,34 \mathrm{kN} / \mathrm{m}^{3} \\ c_{2} & 0,697 & 61 & 15,53 \\ c_{4} & 0,612 & 82 & 16,36 \\ c_{7} & 0,558 & 95,4 & 16,91\end{array}$

Fig. 4 Déformations volumiques totales sous compression isotrope

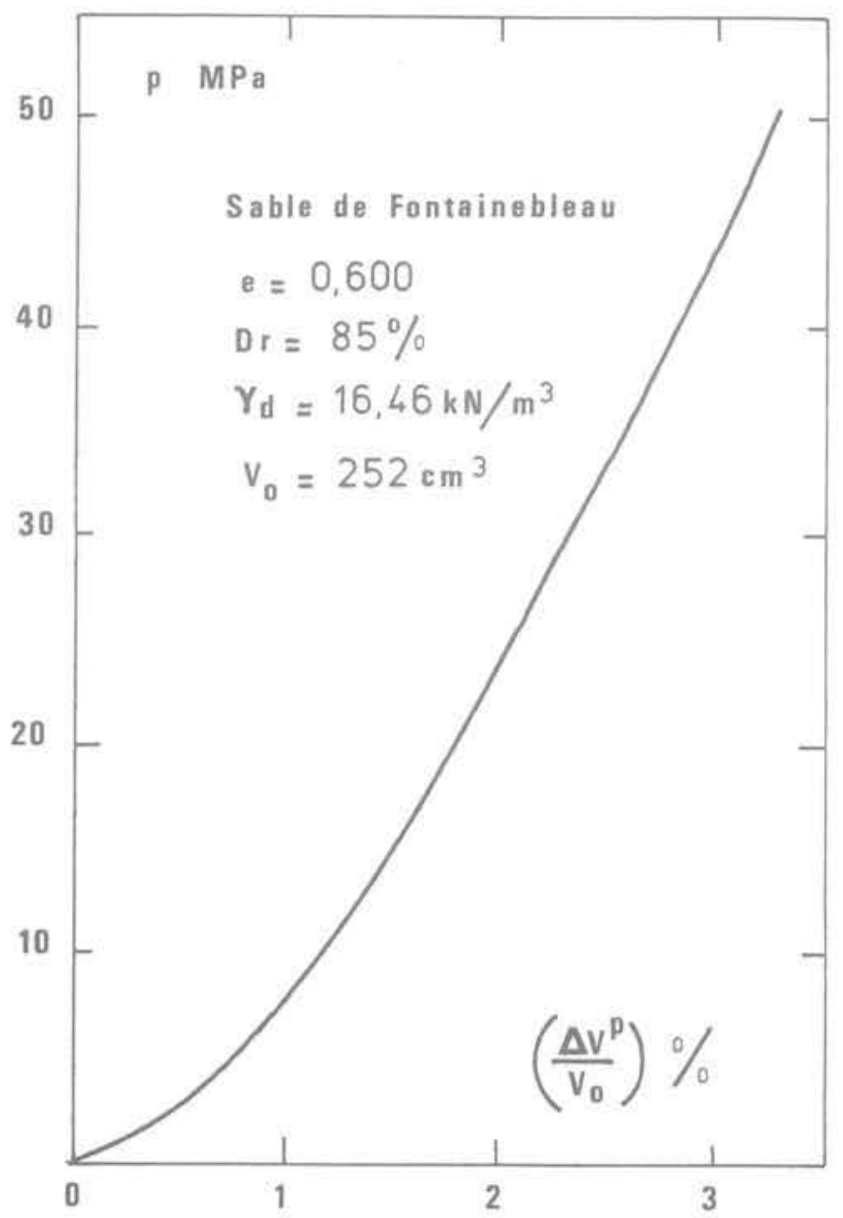

Fig. 5 Déformation volumique irréversible sous contrainte isotrope

\subsection{Chargement cyclique}

Après une première mise en charge, l'effet des cycles induit une densification du matériau par réenchevêtrement géométrique de sa structure.

La figure 6 donne en exemple l'allure caractéristique des cycles d'effort-déformation obtenus lors des essais en termes de contrainte moyenne cyclique et de variation de volume $\Delta \mathrm{V} / \mathrm{V}_{\mathrm{o}}$.

Lorsqu'on observe la réponse du matériau à la décharge, on remarque que sur plusieurs cycles, le diagramme effort-déformation $\left(\mathrm{p}, \Delta \mathrm{V} / \mathrm{V}_{\mathrm{o}}\right)$ reste pratiquement invariable, quelle que soit l'amplitude fixée de la pression isotrope cyclique. Les déformations enregistrées lors de la décharge isotrope d'un matériau pulvérulent restent donc réversibles.

Cette quasi-élasticité du matériau à la décharge, vis-à-vis du comportement à la charge, a été reconnue par les essais de chargements radiaux d'El Sohby (1969). Le trajet de chargement isotrope n'est autre qu'un cas particulier de ce type de chargement.

Les déformations irréversibles cumulées au cours des chargements cycliques isotropes sont développées lors des recharges plus importantes, c'est-à-dire lorsque la contrainte hydrostatique appliquée au matériau croît. Ces cycles de chargement successifs mettent en évidence une différence de réponse entre recharge et décharge, et l'existence d'une boucle d'hystérésis traduisant une dissipation de l'énergie. Les déformations irréversibles apparaissent nettement au cours des premiers cycles. Par la suite, elles ne sont plus détectables au cours d'un cycle qui semble fermé, mais on peut les mettre en évidence par l'enregistrement de deux cycles, suffisamment espacés dans le temps.

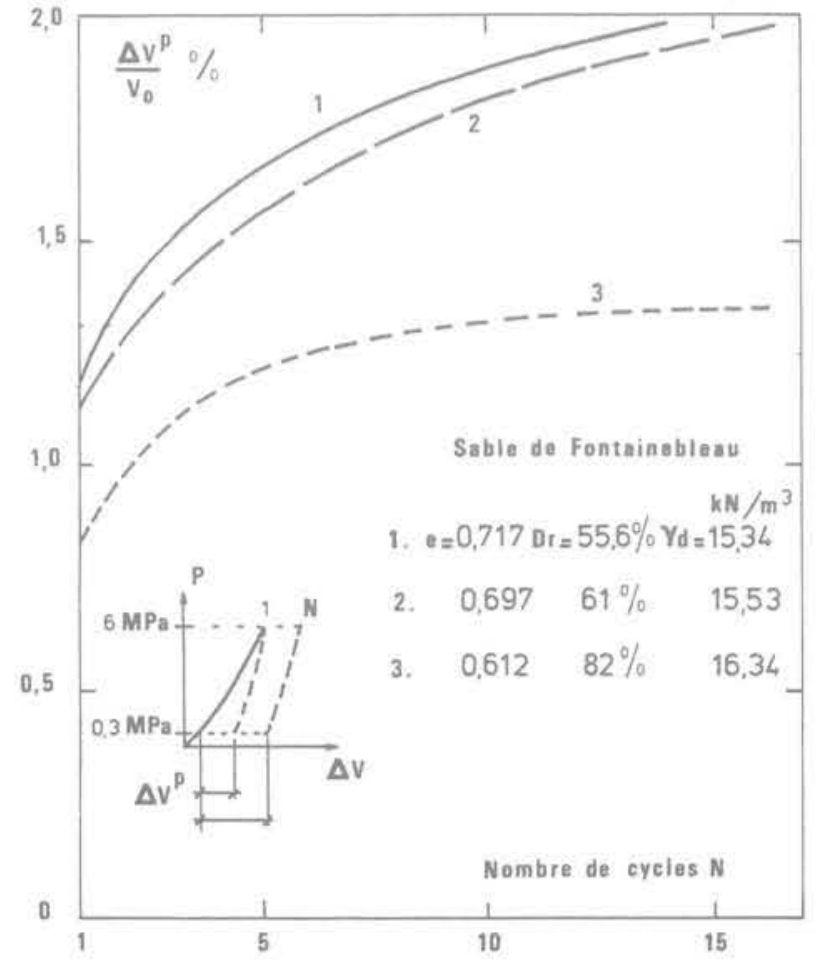

Fig. 7 Déformations volumiques irréversibles en fonction du nombre de cycles $N$ 


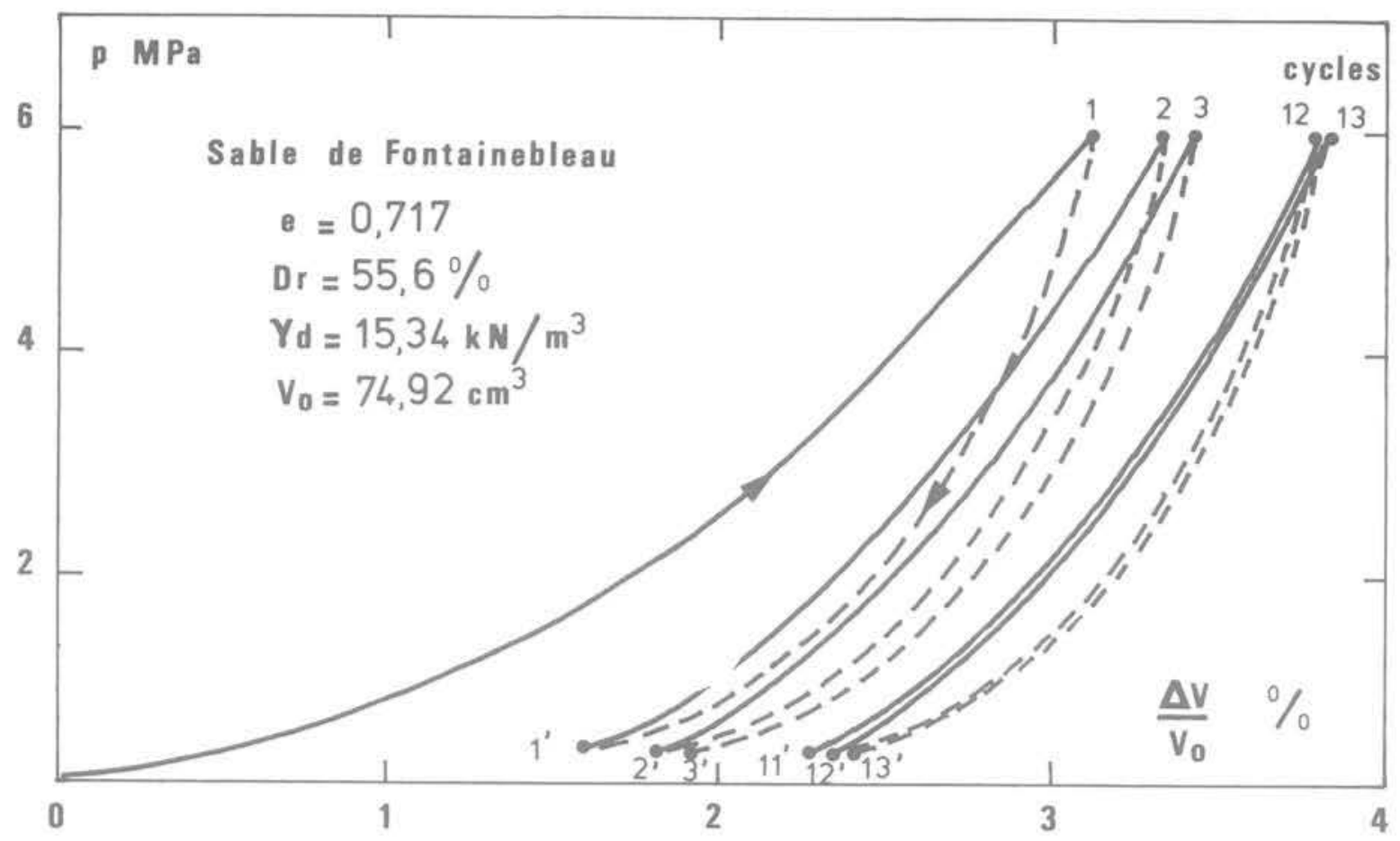

Fig. 6 Déformations volumiques sous compressions isotropes cycliques

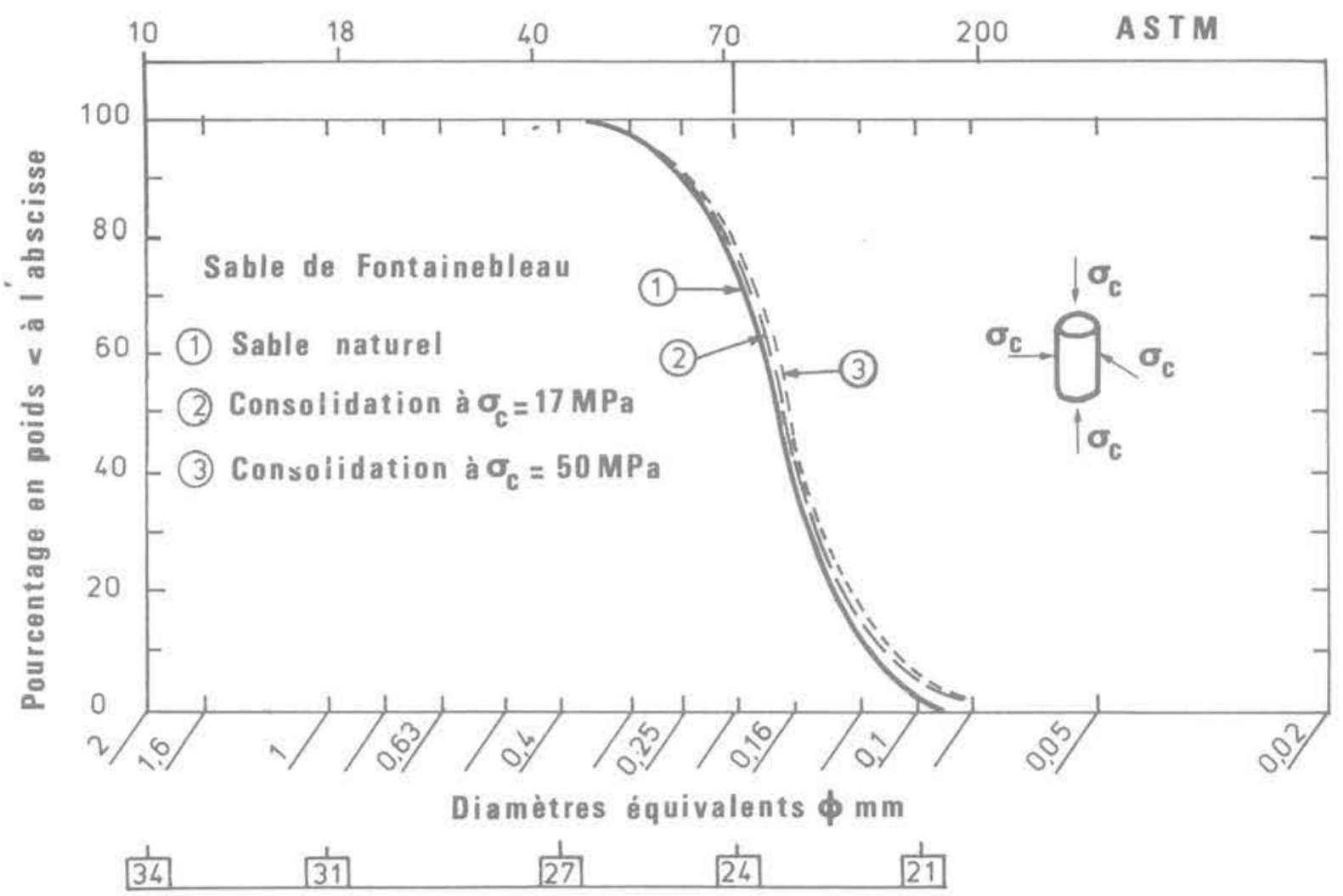

module AFNOR

Fig. 8 Granulométries du sable avant essais et après compressions isotropes jusqu'à $p=50 \mathrm{MPa}$

Les variations de volume irréversibles $\Delta V^{P} / V_{0}$ par rapport au volume initial sont données sur la figure 7 en fonction du nombre de cycles. La déformation irréversible tend asymptotiquement avec le nombre de cycles vers une limite supérieure qui se stabilise au bout d'un certain nombre de cycles variable selon la densité initiale et l'amplitude de la contrainte moyenne cyclique. Pour un sable dense soumis à une contrainte hydrostatique de $6 \mathrm{MPa}$, la diminution de volume se stabilise à une valeur de $0,85 \%$ du neuvième cycle 
(valeur supérieure de $40 \%$ à celle obtenue au premier cycle. Pour une contrainte de $17 \mathrm{MPa}$, la diminution de volume croit rapidement avec le nombre de cycles $\left(\Delta \mathrm{V}^{\mathrm{P}} / \mathrm{V}_{\mathrm{O}}=2,5 \%\right.$ au $16^{\circ}$ cycle $)$ et la tendance à long terme n'apparaît qu'au bout d'un nombre de cycles important, de l'ordre de quelques dizaines.

Le comportement observé est assimilable au phénomène d'adaptation pouvant être considérée sinon comme une borne précise des déformations permanentes, du moins comme un taux d'évolution décroissant avec le nombre de cycles.

Les déformations irréversibles pour le matériau lâche sous contrainte isotrope montrent le rôle essentiel joué par le mécanisme de serrage. La compressibilité irréversible diminue avec l'augmentation de la pression.

L'écrasement des grains sous contrainte isotrope est peu significatif comme le montre la figure 8 qui compare les granulométries du matériau avant essais et après compressions isotropes jusqu'à $p=50 \mathrm{MPa}$.

\section{Comportement lors des essais drainés}

Une même densité relative $\mathrm{Dr}=95 \%$ à la mise en place permet de comparer les résultats des divers essais effectués sur les trois matériaux:

- Sable de Fontainebleau $\gamma_{d}=16,9 \mathrm{kN} / \mathrm{m}^{3}$.

- Sable de Loire

$17,0 \mathrm{kN} / \mathrm{m}^{3}$

- Sable de Seine

$18,4 \mathrm{kN} / \mathrm{m}^{3}$

qui sont donnés sur les figures 9,10 et 11 .

Toutes les courbes $\left(\varepsilon_{\mathrm{q}}, \eta\right)$, quels que soient le matériau et la pression de confinement, présentent une partie initiale linéaire suivie d'un changement de pente progressif avec une concavité du côté des abscisses $\varepsilon_{\mathrm{q}}$. Suivant la pression de confinement, l'augmentation de la distorsion fait apparaître ensuite soit un léger pic suivi d'un palier, soit un palier non précédé d'un pic, soit encore un écrouissage non linéaire. La résistance au cisaillement sera définie comme la valeur maximale du niveau de contrainte $\eta_{\max }=\eta_{1}$ que peut supporter l'échantillon au cours de l'écrasement.

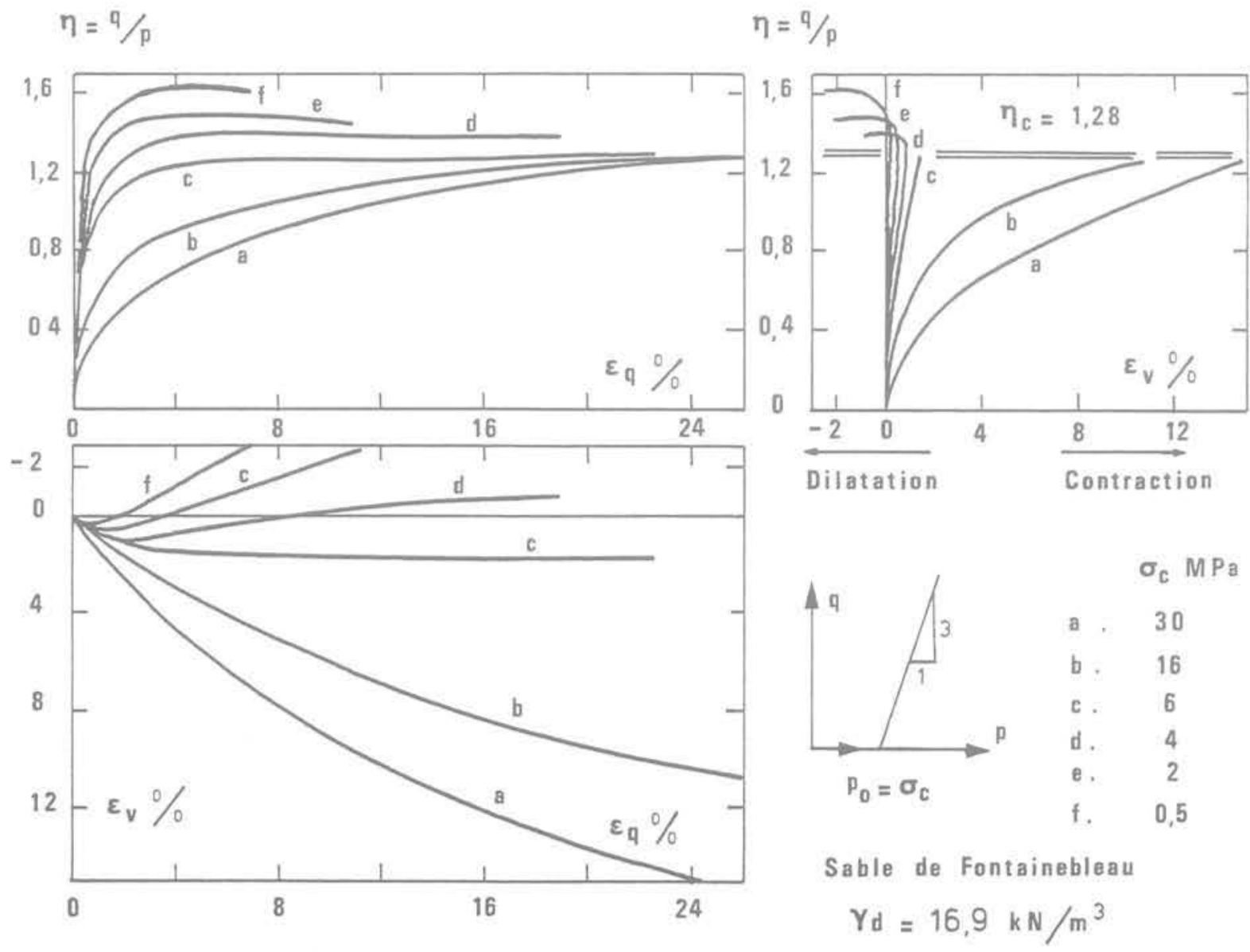

Fig. 9 Compression triaxiale conventionnelle à différents confinements 


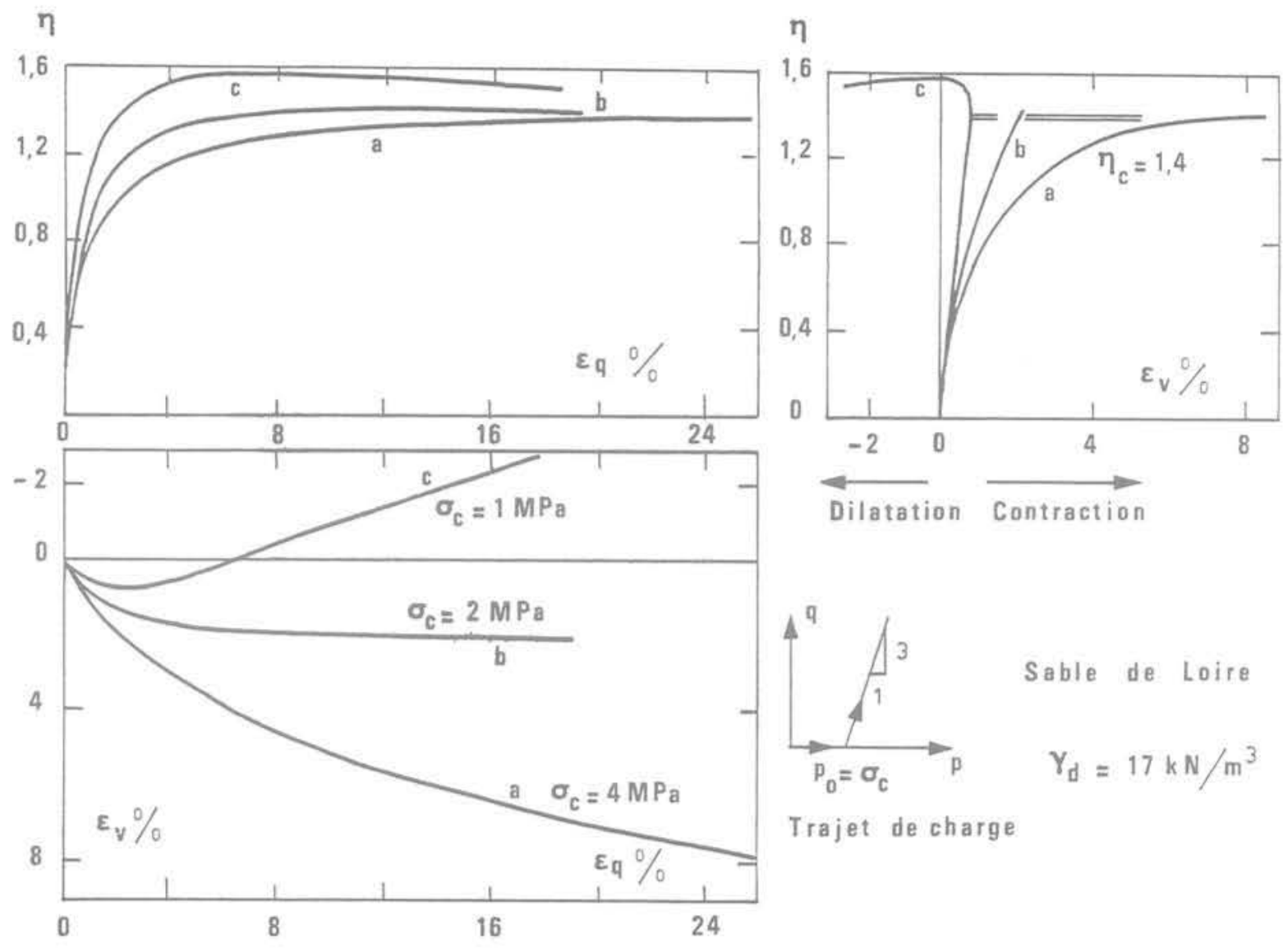

Fig. 10 Sable de Loire sous compression axisymétrique à confinement constant
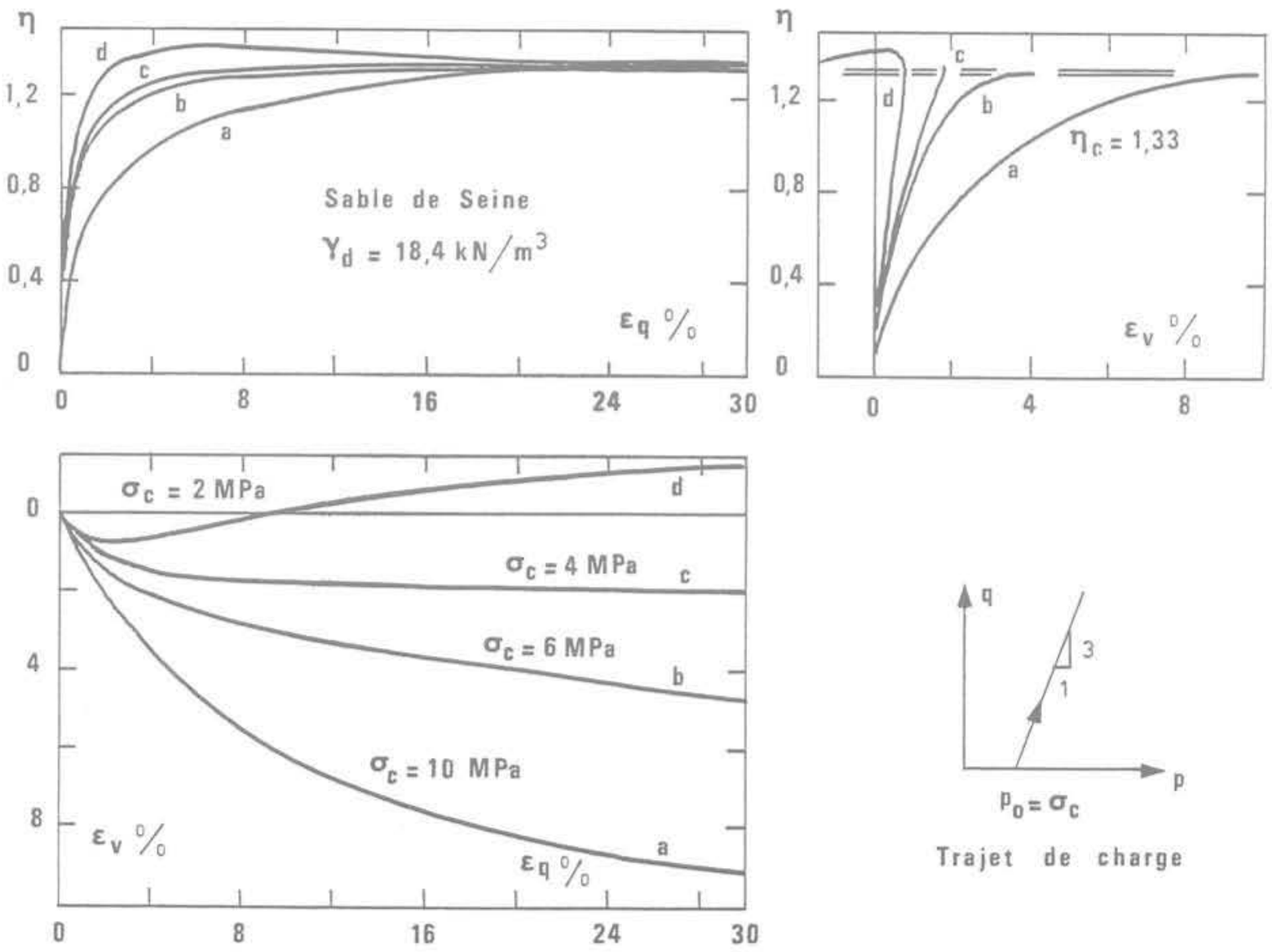

Trajet de charge

Fig. 11 Sable de Seine sous compression axisymétrique à confinement constant 


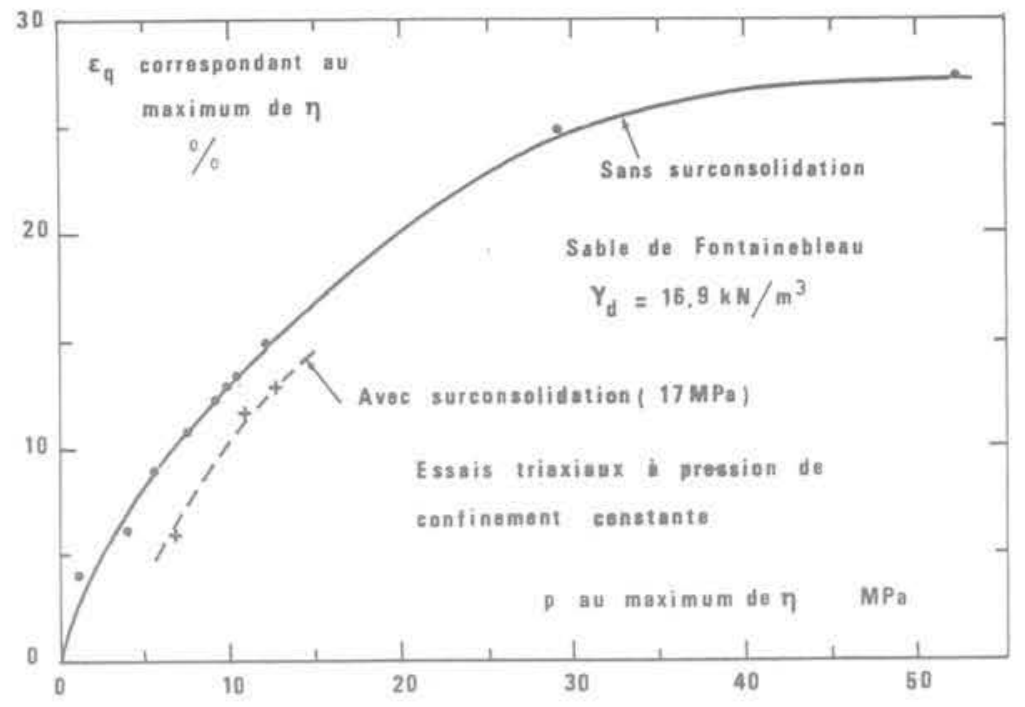

Fig. 12 Distorsions $\epsilon_{q}$ à la résistance maximale

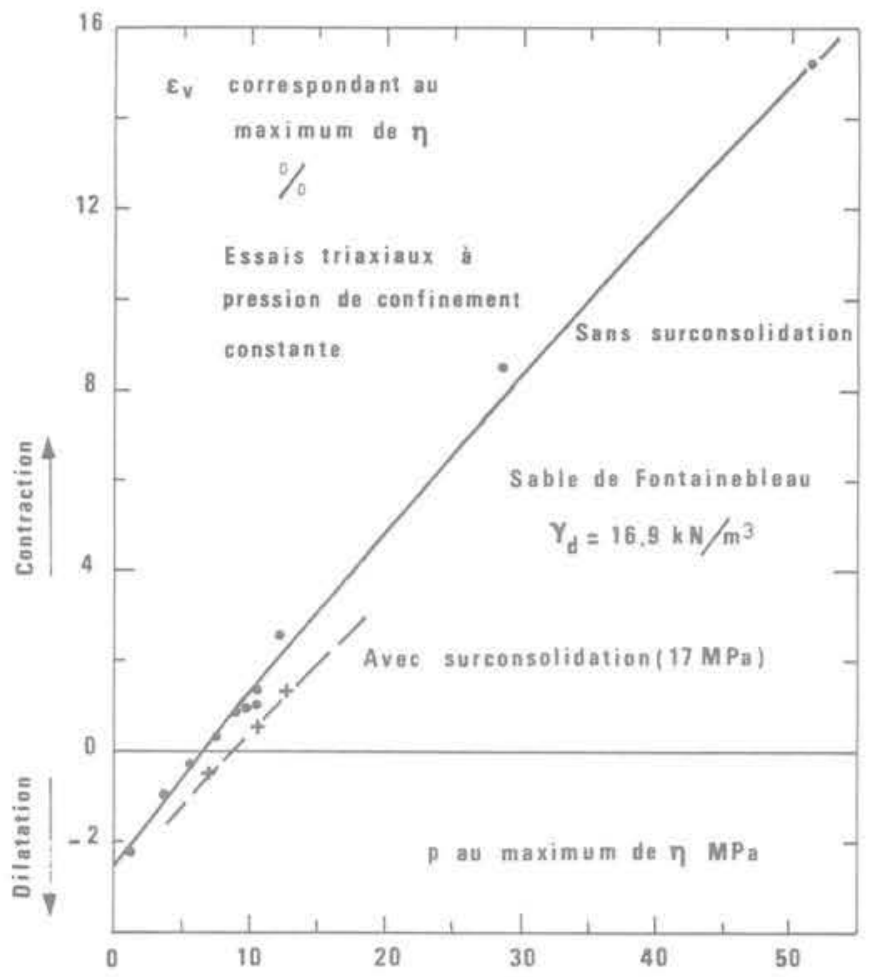

Fig. 13 Déformations volumiques $\epsilon_{v}$ à la résistance maximale

L'abscisse $\varepsilon_{\mathrm{q}}$ correspondant au maximum de $\eta=\eta_{\mathrm{t}}$ augmente sensiblement avec la pression $\sigma_{c}$ où avec la contrainte moyenne $\left(p=3 \sigma_{c} /\left(3-\eta_{\max }\right)\right.$ pour le trajet triaxial conventionnel).

La figure 12 suggère un palier asymptotique de la distorsion $\varepsilon_{q}$ lorsque la contrainte moyenne $p$ augmente.

La figure 13 montre dans le diagramme $\left(p, \varepsilon_{v}\right)$ une augmentation progressive de la compressibilité volumique en fonction de la pression de confinement i.e. de la contrainte moyenne $\mathrm{p}$. On peut ainsi distinguer deux types de comportement:

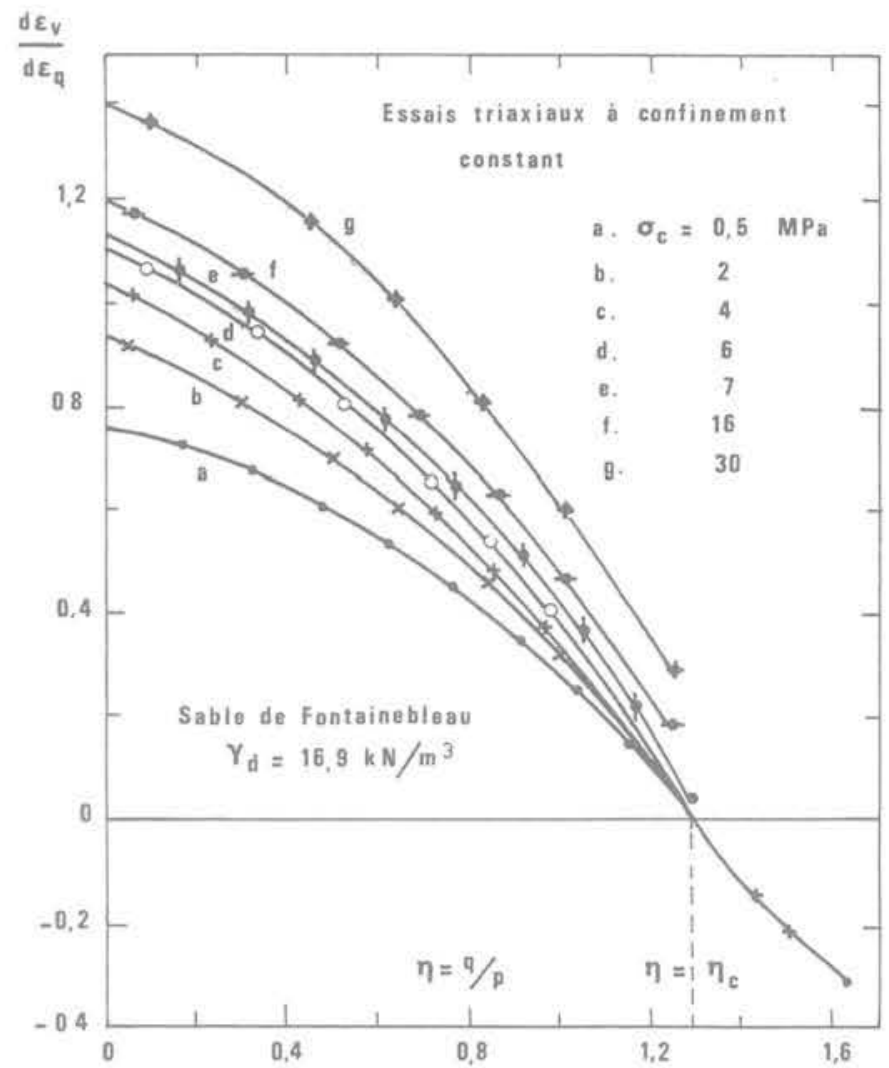

Fig. 14 Variation du taux de dilatance au cours des essais en compression triaxiale

- A faible confinement, le chargement entraîne une contraction volumique suivie d'une dilatation continue jusqu'à l'écoulement du matériau.

- A confinement plus important, seule une contraction continue apparait au cours du chargement jusqu'à l'écoulement plastique.

Pour les trciẽ sables étudiés, le niveau de contrainte correspondant au début de la phase de dilatance Iorsqu'elle existe au cours d'une compression triaxiale - est stable. Ce niveau de contrainte $\eta=\eta_{\mathrm{c}}$, associé à une vitesse de déformation volumique nulle $d \varepsilon_{v}=0$ définit un angle de frottement caractéristique $\varphi_{c}$ qui 


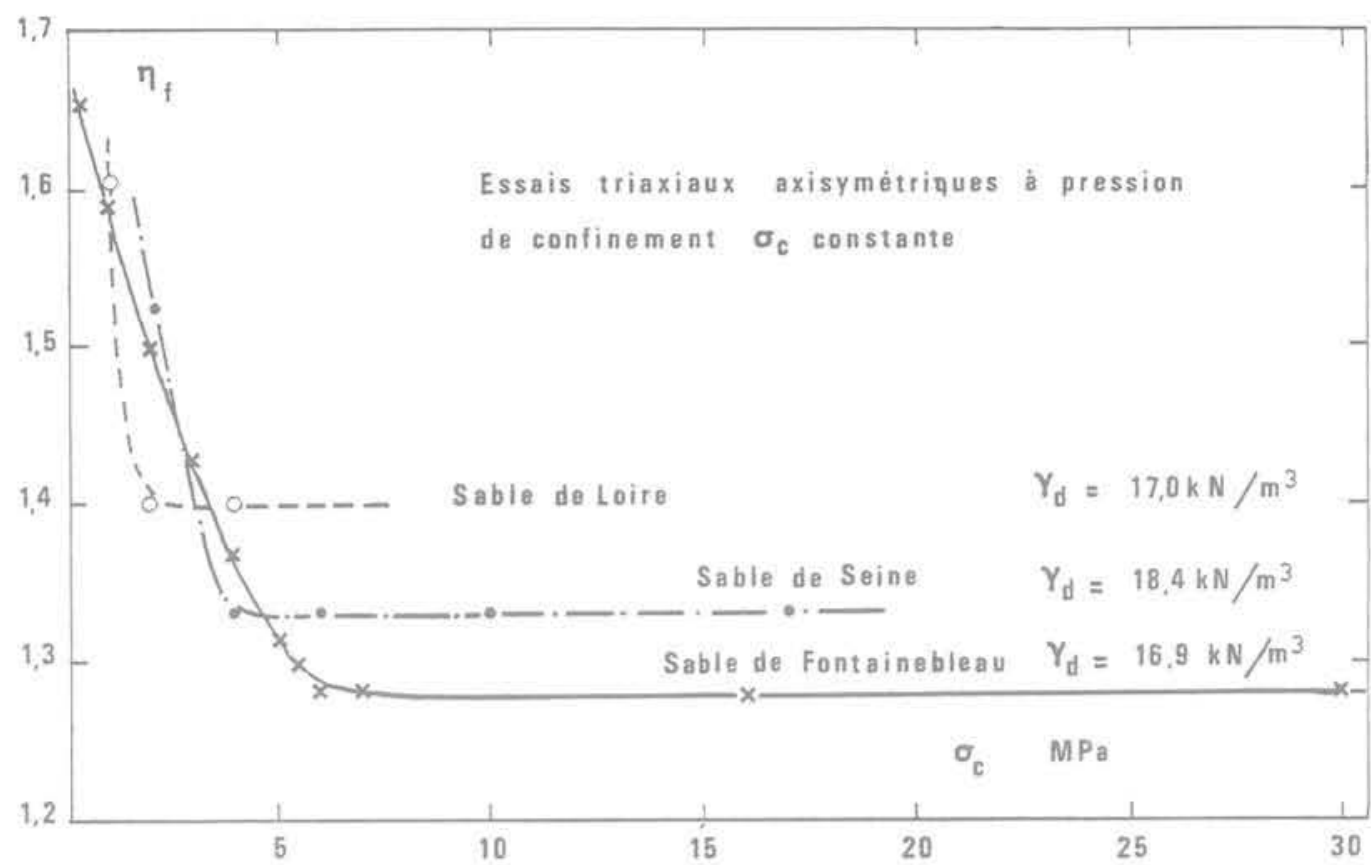

Fig. 15 Résistance au cisaillement $\eta_{f}$ en fonction de la pression de confinement constante

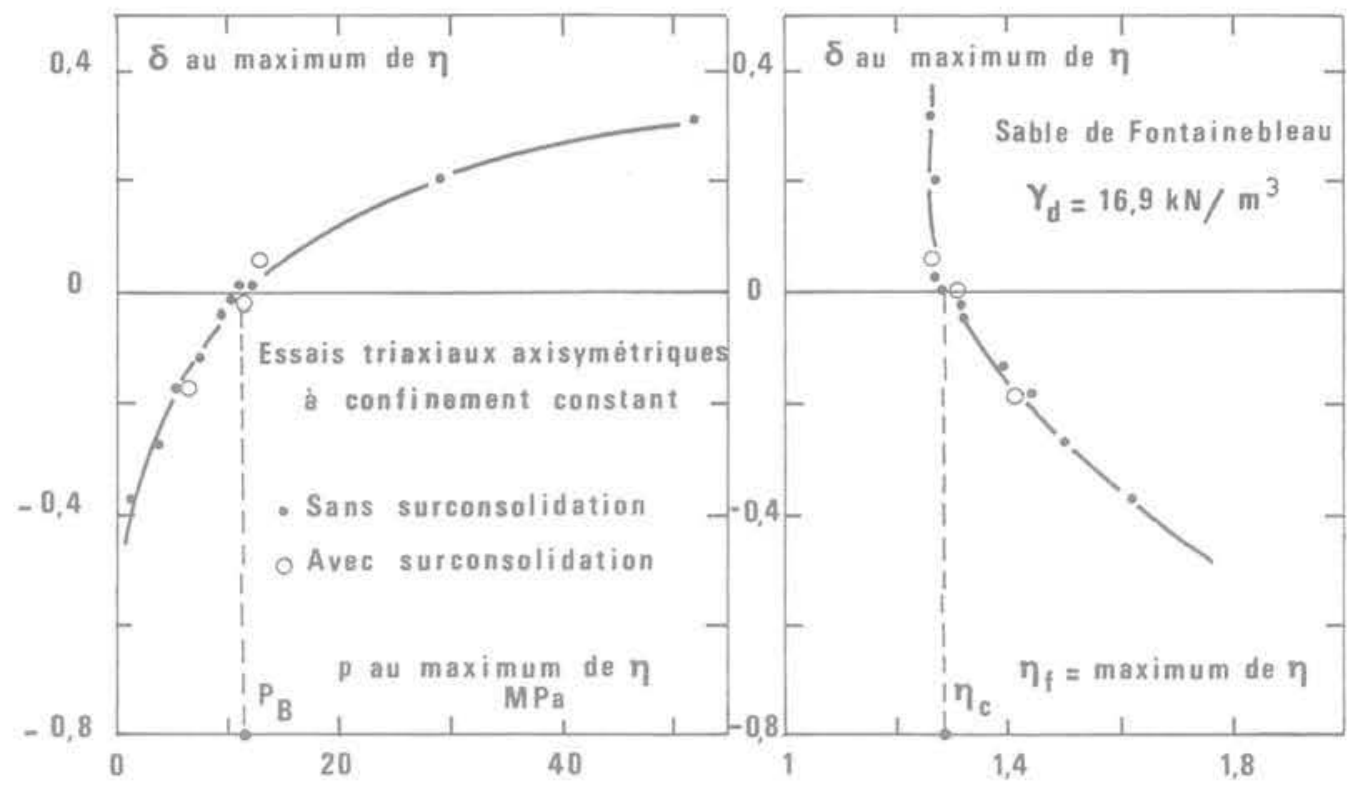

Fig. 16 Evolution de la vitesse de dilatance $\delta=d \epsilon_{v} / d \epsilon_{q}$ à l'écoulement plastique $\eta_{f}=(q / p)$ maximum

détermine le seuil d'enchevêtrement des grains $\varphi_{c}=\operatorname{Arcsin}\left(3 \eta_{c} /\left(6+\eta_{c}\right)\right)$ [Luong 1978].

La figure 14 montre la variation de la pente de la courbe $\left(\varepsilon_{\mathrm{q}}, \varepsilon_{\mathrm{v}}\right)$ au cours du chargement, donnée par le rapport $\mathrm{d} \varepsilon_{\mathrm{v}} / \mathrm{d} \varepsilon_{\mathrm{q}}$ à différents niveaux déviatoriques de contrainte $\eta=q / p$ pour diverses pressions de confinement. L'influence prépondérante de la pression de confinement $\sigma_{c}$ sur l'accroissement relatif de la compressibilité volumique par rapport à la distorsion se traduit par une augmentation sensible de la pente à l'origine de la courbe $\left(\varepsilon_{\mathrm{q}}, \varepsilon_{\mathrm{v}}\right)$ avec le confinement $\sigma_{\mathrm{c}}$.

La figure 15 présente l'allure des courbes $\left(\sigma_{c}, \eta_{t}\right)$ des trois sables étudiés : la résistance au cisaillement $\eta_{t}$ décroît avec la pression de confinement $\sigma_{c}$ et tend vers une limite pratiquement constante et égale à $\eta_{c}$ valeur caractéristique.
L'évolution du mode d'écoulement peut être décrite par la vitesse de dilatance à l'écoulement plastique $\left(\mathrm{d} \varepsilon_{\mathrm{v}} / \mathrm{d} \varepsilon_{\mathrm{q}}\right)_{\mathrm{t}}=\delta_{\mathrm{f}}$ correspondant au niveau déviatorique maximal $\eta_{t}$. La figure 16 met en évidence la relation entre ces deux paramètres qui caractérisent l'écoulement plastique du matériau en fonction de la contrainte moyenne $\mathrm{p}$ : la vitesse de dilatance augmente continûment avec la contrainte moyenne. Elle s'annule et change de signe à partir de $p=p_{B}$.

- Pour $p<p_{\mathrm{B}}$, le taux de dilatance $\delta_{\mathrm{r}}$ à la rupture est négatif, ce qui traduit un comportement dilatant du matériau à l'écoulement plastique.

- Pour $p>p_{B}$, le taux de dilatance $\delta$, à la rupture est positif, le matériau est contractant à l'écoulement plastique. $\delta$, augmente continûment avec la contrainte moyenne tandis que $\eta_{t}$ garde une valeur pratiquement stable $\eta_{\mathrm{t}}=\eta_{\mathrm{c}}$ à partir de $\mathrm{p}=\mathrm{p}_{\mathrm{B}}$. 

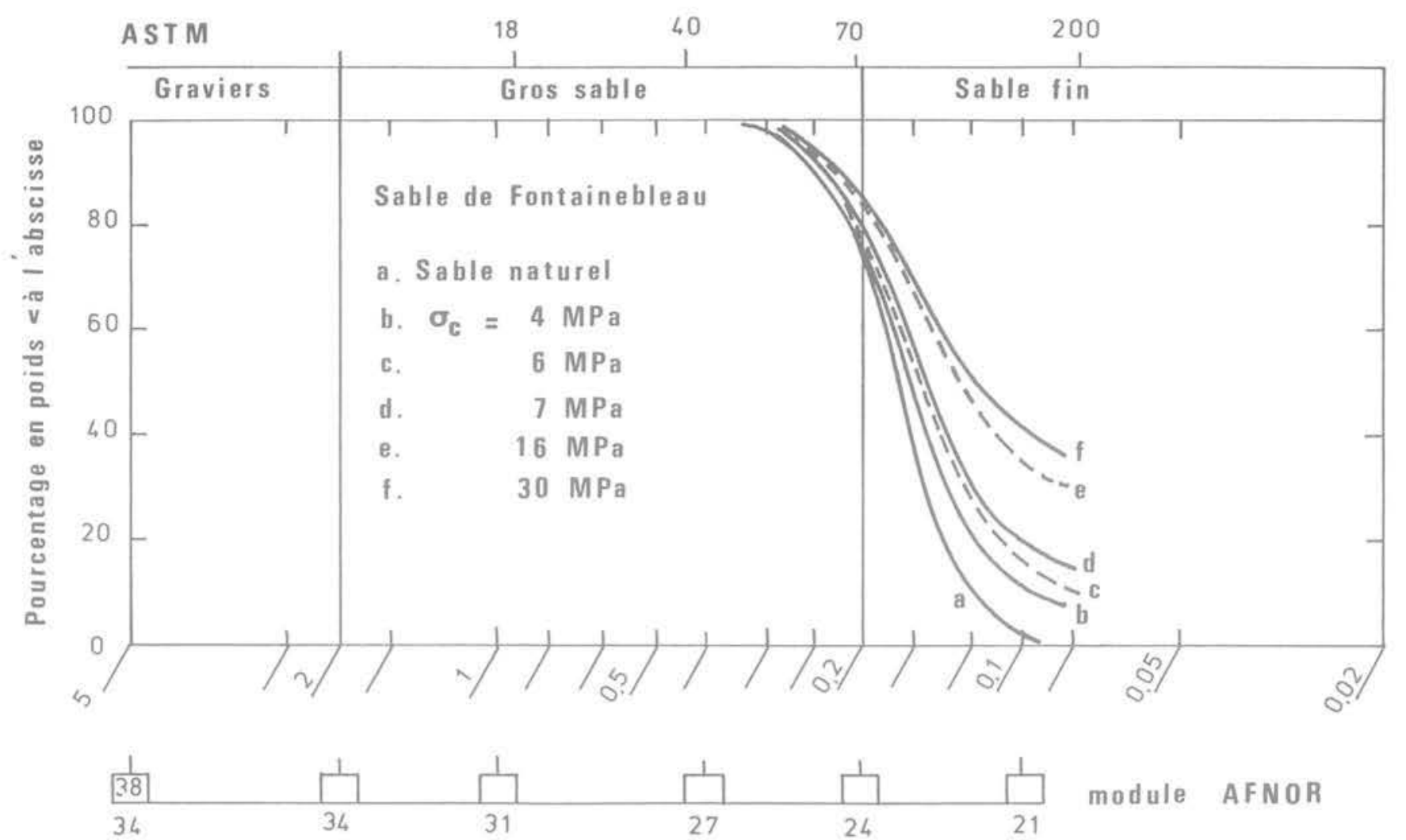

Fig. 17 Granulométrie avant et après essais triaxiaux

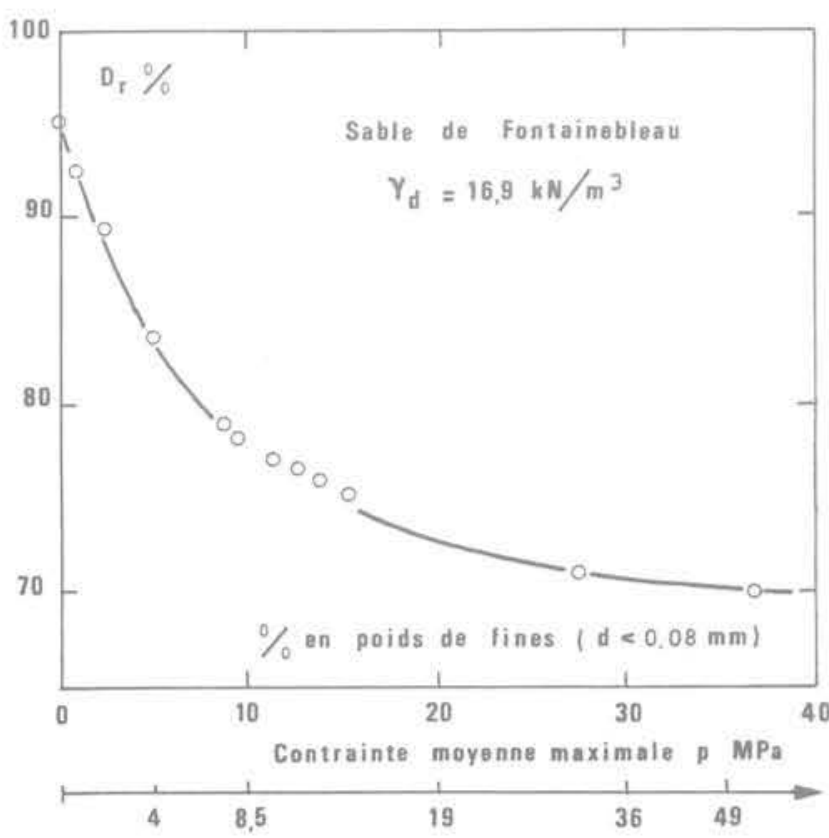

Fig. 18 Diminution de la densité relative en fonction de l'attrition des grains

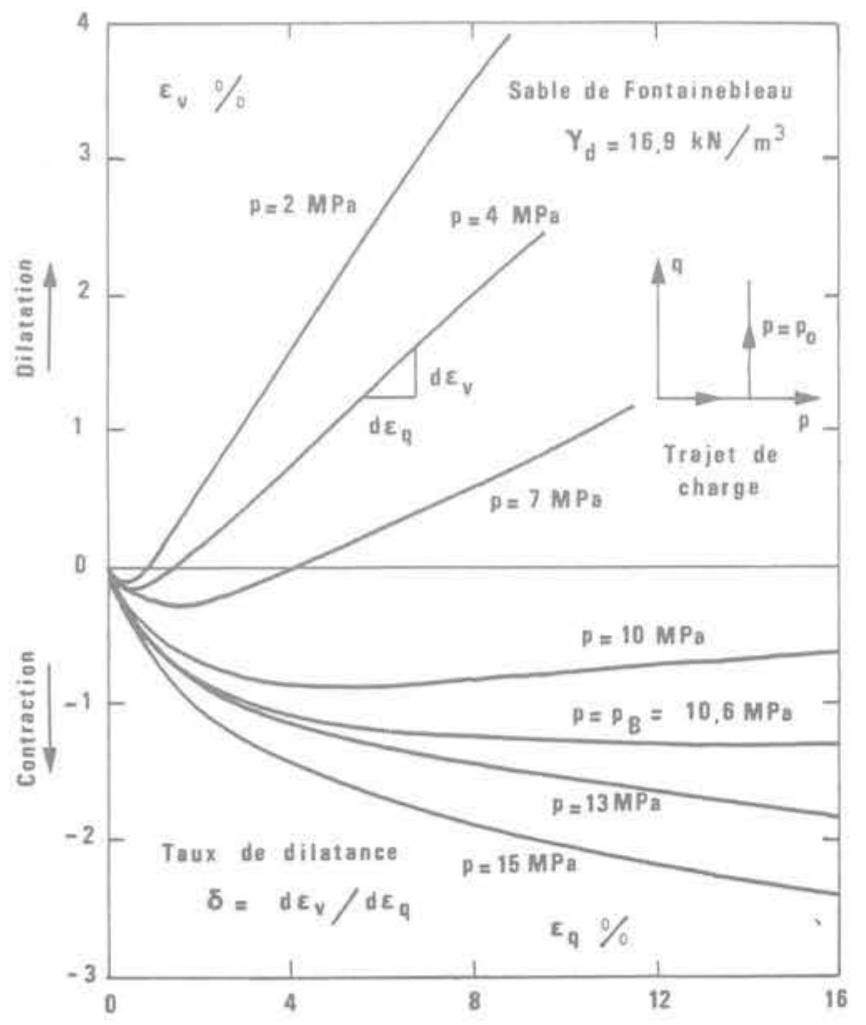

Fig. 19 Courbes distorsion-déformation volumique en compression triaxiale à contrainte moyenne constante 

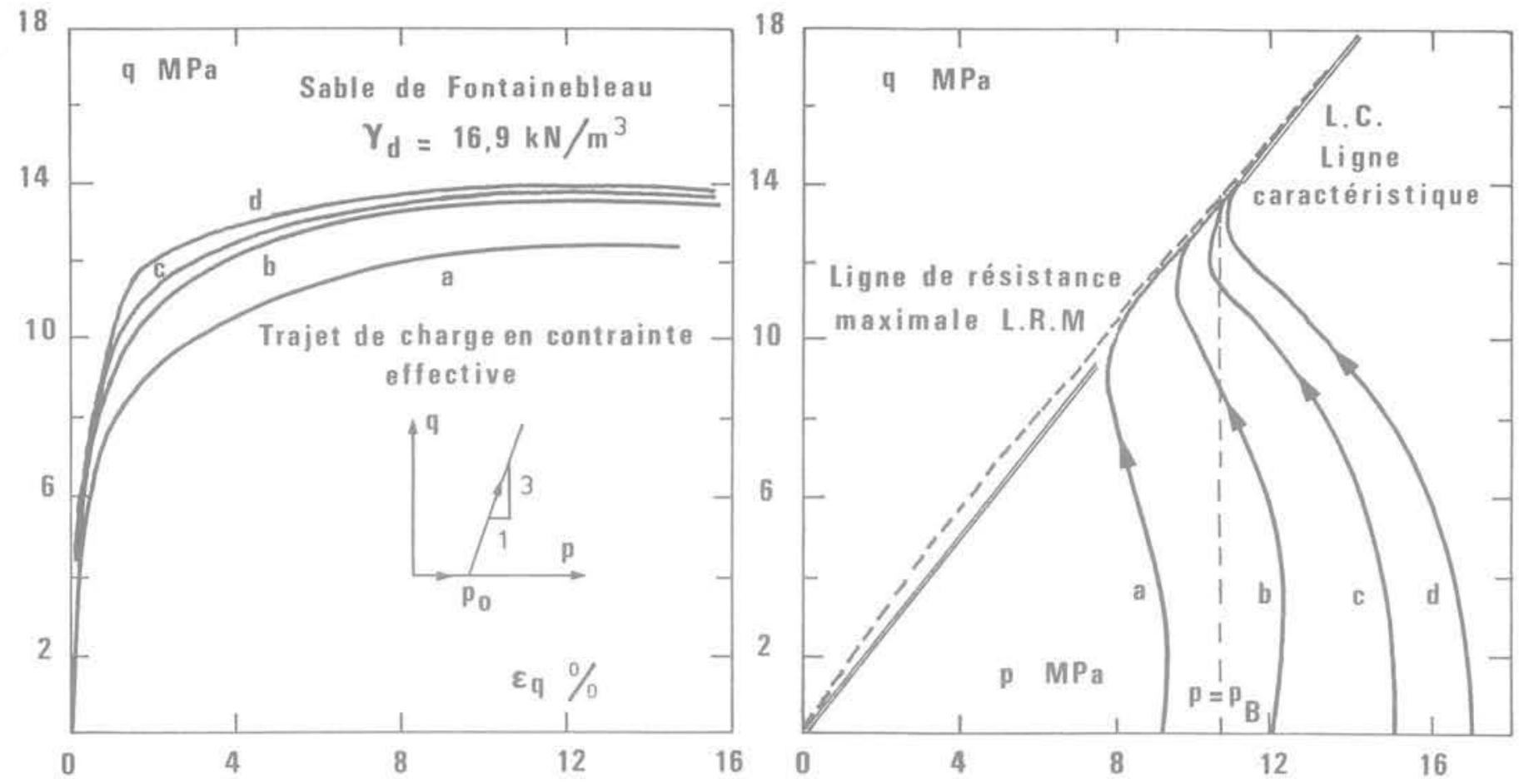

Fig. 20 Sable dense de Fontainebleau en cisaillement axisymétrique non drainé

La contrainte moyenne $\mathrm{p}_{\mathrm{B}}$ définit donc pour ces matériaux un seuil de comportement dilatant à l'écoulement plastique avec un palier sur la courbe $\left(\varepsilon_{v}\right.$, $\left.\varepsilon_{q}\right)$ i.e. $\delta_{t}=\left(\frac{d \varepsilon_{v}}{d \varepsilon_{q}}\right)_{t}=0$.

Les essais effectués ont donnè :

$\mathrm{p}_{\mathrm{B}}=10,6 \mathrm{MPa}$ pour le sable de Fontainebleau,

$3,7 \mathrm{MPa}$ pour le sable de Loire,

7,2 MPa pour le sable de Seine.

La figure 17 indique une profonde modification du diamètre des grains due au cisaillement drainé sous des pressions de confinement élevées, qui tend à rendre la granulométrie de plus en plus étalée. On constate que les essais modifient beaucoup plus le $d_{10}$ que le $\mathrm{d}_{60}$. Après l'essai à $\sigma_{\mathrm{c}}=6 \mathrm{MPa}$ soit $\mathrm{p}_{\max }=\mathrm{p}_{\mathrm{B}}, \mathrm{d}_{10}$ est passé de $0,125 \mathrm{~mm}$ à $0,08 \mathrm{~mm}$ et $d_{60}$ de $0,17 \mathrm{~mm}$ à $0,14 \mathrm{~mm}$. Pour les pressions de confinement $\sigma_{c}$ supérieures à $6 \mathrm{MPa}, \mathrm{d}_{10}$ reste nettement inférieur à $0,08 \mathrm{~mm}$. Le pourcentage des grains de diamètre inférieur à $0,08 \mathrm{~mm}$ est une fonction croissante de la contrainte moyenne p maximale appliquée au matériau et modifie de façon sensible la densité relative du matériau (figure 18).

\section{Compression triaxiale conventionnelle}

Le trajet de chargement à contrainte moyenne $p$ constante est obtenu à l'appareil triaxial en faisant varier simultanément et linéairement la pression de confinement $\sigma_{c}$ et le déviateur de contrainte $q=3 p-$ $3 \sigma_{\mathrm{c}}$ à partir d'un état de contrainte isotrope $\sigma_{1}=\sigma_{2}=$ $\sigma_{3}=p$.

Les résultats obtenus sont analogues à ceux obtenus sur le trajet triaxial conventionnel à confinement constant. Au cours des essais effectués sous contrainte moyenne $p$ constante inférieure à $10,6 \mathrm{MPa}$, le comportement du matériau est d'abord contractant puis dilatant. Lorsque $\mathrm{p}$ est supérieure à 10,6 MPa, on n'observe plus de dilatation volumique du matériau qui s'écoule avec diminution de volume. Avec la valeur de $p_{\mathrm{B}}=10,6 \mathrm{MPa}$, le comportement du sable est caractérisé par une contraction volumique suivie d'un écoulement plastique à taux de déformation volumique nul $\mathrm{d} \varepsilon_{\mathrm{v}}=0$ avec un palier à partir de $\varepsilon_{\mathrm{q}}=8 \%$ (figure 19).

\section{Compression triaxiale}

Les essais triaxiaux en condition non drainée imposent une condition en déformation de non variation de volume global de l'échantillon $(d V=0)$. Si l'échantillon est saturé, la tendance à la contraction ou à la dilatation du matériau granulaire sera compensée par une augmentation ou une diminution de la pression interstitielle u dont la mesure directe permet de préciser la réponse du matériau en contrainte effective correspondant à chaque type de comportement du squelette solide dans le plan (p, q) [figure 20].

Les essais non drainés ont été effectués sur un sable dense de Fontainebleau de même poids volumique $\gamma_{\mathrm{d}}=16.9 \mathrm{kN} / \mathrm{m}^{3}$ que pour les essais de cisaillement drainé. Le matériau est soumis à une consolidation isotrope $p=9,1 ; 12,0 ; 15,0 ; 17,0 \mathrm{MPa}$, suivie d'une compression triaxiale en condition non drainée.

Au cours du chargement, on constate d'abord une augmentation de la pression des pores indépendamment de la contrainte moyenne initiale $p_{0}$. La déformation de distorsion subie par l'échantillon reste dans ce cas relativement limitée. Lorsque le niveau déviatorique de contrainte $\eta$ s'approche suffisamment de la valeur caractéristique $\eta_{c}$, le taux de variation de la pression interstitielle commence à décroître jusqu'à la valeur nulle $\dot{\mathrm{u}}=0$. On peut alors observer deux types de comportement suivant la valeur de la contrainte moyenne effective $p^{\prime}$ atteinte au cours de l'essai : 
- diminution de la surpression interstitielle jusqu'à l'écoulement plastique à un niveau de contrainte $\eta$ plus élevé $\eta_{\mathrm{t}}>\eta_{\mathrm{c}}$,

- écoulement plastique sans variation de la pression des pores. Pour $\mathrm{p}_{\mathrm{o}}=17 \mathrm{MPa}$, un palier de la courbe $\left(\varepsilon_{q}, u\right)$ apparaît sans être précédé d'une diminution de la surpression interstitielle: $\eta_{\mathrm{t}}$ mobilisé reste pratique égal à $\eta_{c}$.

Dans le plan $(p, q)$, le trajet en contrainte effective présente d'abord une concavité vers les abscisses décroissantes, puis change de concavité au voisinage de la ligne de pente $q / p=\eta_{c}$.

Dès que l'état de contrainte effective permet l'écoulement plastique, le trajet en contrainte effective suit la ligne de résistance maximale et converge au point d'abscisse $p_{B}$ qui est l'unique point qui vérifie la condition de non variation de volume à l'écoulement plastique se traduisant par:

$$
\mathrm{p}=\mathrm{p}_{\mathrm{B}} \quad \mathrm{d} \varepsilon_{\mathrm{v}}=0 \quad \text { à } \quad \eta=\eta_{\mathrm{f}}=\eta_{\mathrm{c}} \text {. }
$$

\section{Synthèse des résultats}

Les différents essais présentés mettent en évidence l'importance de l'évolution micro-structurale des milieux granulaires dans la description de leur comportement mécanique sous contraintes élevées: l'attrition des grains élémentaires, qui n'est pas directement un phénomène lié à la structure globale,

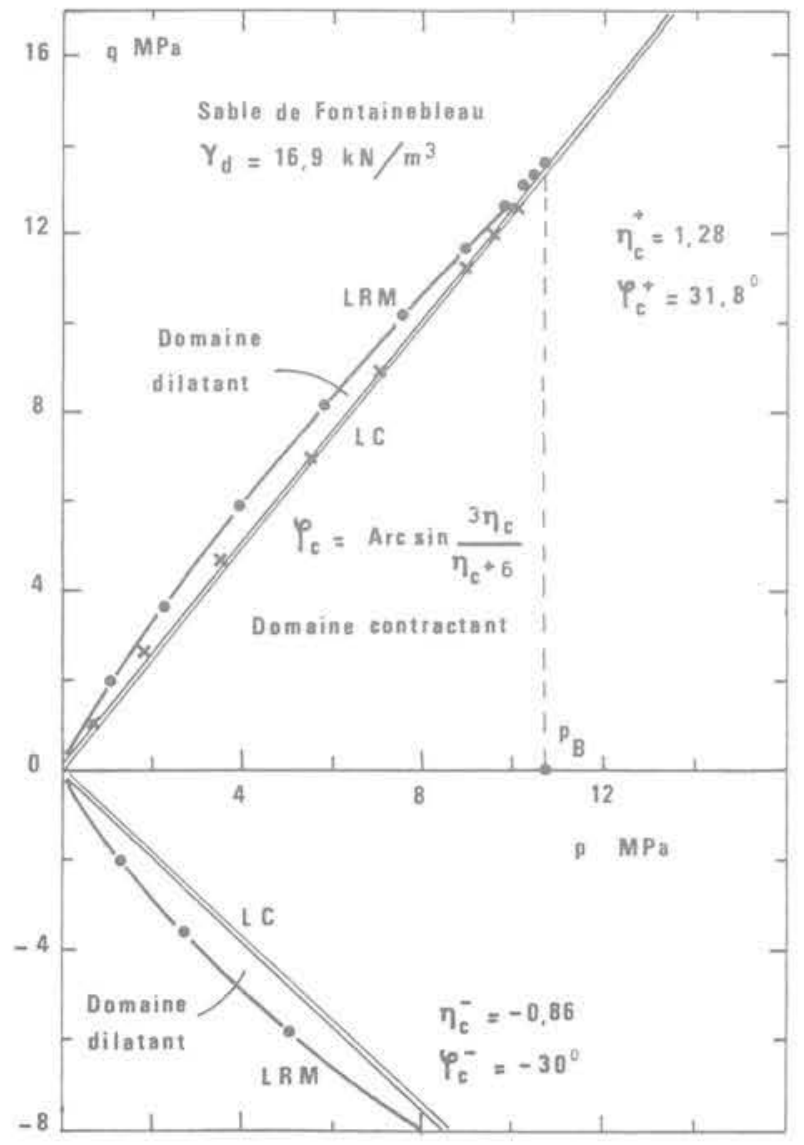

Fig. 21 Domaines contractant et dilatant du sable de Fontainebleau est cependant susceptible d'entraîner des déformations importantes du matériau en activant le mécanisme de réarrangement des grains de caractère purement géométrique.

Du point de vue du comportement global, le phénomène important à décrire est l'évolution immédiate de l'état de volume du milieu, i. e. la contractance ou la dilatance, développée au cours du chargement. Ainsi cette évolution est étroitement associée aux propriétés de génération de la pression interstitielle dont le développement conditionne la stabilité des ouvrages de génie civil.

Dans l'espace des contraintes $(p, q)$ pour des faibles contraintes moyennes, le seuil caractéristique $\eta_{c}$ sépare deux types de comportement rhéologique : contractant dans le domaine subcaractéristique délimité par les lignes caractéristiques LC et dilatant dans le domaine surcaractéristique jusqu'à la ligne de résistance maximale LRM (figure 21).

La contrainte moyenne $p_{B}$ définit le seuil de comportement dilatant du matériau granulaire à l'écoulement plastique, compatible avec son état caractéristique et associé aux propriétés suivantes :

- La vitesse de dilatance à l'écoulement plastique est. nulle

$$
\delta_{1}=\left(d \varepsilon_{v} / d \varepsilon_{q}\right)_{t}=0 .
$$

- Le niveau de contrainte maximal supporté par le matériau définit un angle de frottement interne égal au frottement caractéristique

$$
\eta_{\mathrm{f}}=\eta_{\mathrm{c}} \Longrightarrow \varphi_{\mathrm{t}}=\varphi_{\mathrm{c}} \text {. }
$$

Pour les essais tels que la contrainte moyenne p est supérieure à $\mathrm{p}_{\mathrm{B}}$, les résultats indiquent que le sable subit une contractance continue jusqu'à l'écoulement plastique: l'attrition surabondante des grains augmente sensiblement la compressibilité volumique.

Un comportement analogue a été constaté [17] au cours des essais en compression triaxiale sur le sable de "Chattahoochee River". Ces résultats touchent à des propriétés générales et doivent pouvoir être acceptés pour d'autres matériaux granulaires tels que les enrochements fragmentés [9] utilisés pour les barrages.

\section{Conclusion}

L'approche proposée a été développée dans le cadre du concept de l'état caractéristique basé sur les mécanismes physiques de déformations réversibles et irréversibles au cours du chargement :

- Serrage de la matrice solide (consolidation).

- Désenchevêtrement de la structure granulaire (dilatance).

- Rupture et/ou attrition des grains conduisant à un seuil de contrainte moyenne $\mathrm{P}_{B}$ appelé limite de comportement dilatant du matériau granulaire au-delà de laquelle la dilatance ne peut plus se manifester.

Les résultats expérimentaux obtenus sur plusieurs sables ont montré que l'angle de frottement interne caractéristique $\varphi_{c}$ associé à une vitesse de dilatance nulle est un paramètre intrinsèque indépendant de la porosité initiale, de la contrainte moyenne et insensible aux modifications de la granularité au cours de l'essai. 


\section{Références bibliographiques}

[ 1] CAMBOU B. (1978), Analyse statistique au niveau de la particule d'un milieu granulaire, Journée de Rhéologie 1978, E.N.T.P.E. Vaulx en Velin.

[ 2] CUNDALL P.A. et STRACK O.D.L. (1979), The development of constitutive laws for soil using the distinct element method, Numerical Methods in Geomechanics, vol. 1, Aachen.

[ 3] DANTU P. (1957), Contribution à l'étude mécanique et géométrique des milieux pulvérulents, Proc. 4th I. C. O.S. M. F. E., London, Vol. 1, 144-148.

[ 4] DERESIEWICZ H. (1958), Mechanics of Granular Matter, Advances in Applied Mechanics, Vol. V1958, Academic Press.

[ 5] HABIB P. et LUONG M.P. (1974), Comportement mécanique des sols squs forts recouvrements, $3^{e}$ Congrés International de Mécanique des Roches, Denver Colorado, U.S.A.

[ 6] LEE K.L. (1969), Particle breakage during highpressure testing, Spec. Session $n^{\circ} 13,7$ th I.C.O.S.M.F.E. (Contributions and discussions on mechanical properties of rockfill and gravel materials), Mexico, 141-153.

[ 7] LEE K. L. et FARHOOMAND I. (1967), Compressibility and crushing of granular soils in anisotropic compression, Can. Geot. J., 4(1), 68-86.

[ 8] LE LONG (1968), Contribution à l'étude des propriétés mécaniques des sols sous fortes pressions, Thèse de Docteur-Ingénieur IMG.

[ 9] LESLIE D.D. (1969), Relationship between shear strength, gradation and index properties of rockfill materials, Spec. Session $n^{\circ} 13,7$ th I.C.O.S.M.F.E.
(Contributions and discussions on mechanical properties of rockfill and gravel materials), Mexico, 201-210.

[10] LEUSSINK H. et BRAUNS J. (1969), Results of recent investigations on shear strength characteristics of granular masses at high pressures, Spec. Session $n^{\circ} 13,7$ th I.C.O.S.M.F.E. (Contributions and discussions on mechanical properties of rockfill and gravel materials), Mexico, 43-50.

[11] LUONG M.P. (1980), Phénomènes cycliques dans les sols pulvérulents, Revue Française de Géotechnique, 10 , pp. 39-53.

[12] LUONG M.P. (1978), État caractéristique du sol, C.R.A.S. Paris, t. 287, Nov. 1978, Série B, 305-307.

[13] MARSAL R.J. (1969), A statistical model for granular materials, Spec. Session $n^{\circ} 13,7$ th I.C.O.S.M.F.E. (Contributions and discussions on mechanical properties of rockfill and gravel materials), Mexico, 115-122.

[14] MINDLIN R.D. (1949), Compliance of elastic bodies in contact, J. of Applied Mechanics, Transactions A.S. M.E. Vol. 71 , pp. $259-268$.

[15] TOUATI A. (1982), Comportement mécanique des sols pulvérulents sous fortes contraintes, Thèse de Docteur-Ingénieur E. N.P. C., Paris, Octobre 1982.

[16] VESIC A.S. et BARKSDALE R.D. (1963), On shear strength of sand at very high pressures. Proceedings Symposium on Laboratory Shear Testing of Soils, Ottawa, A.S.T.M. Spec. Tech. Publ. No 361, pp. 301305 .

[17] VESIC A.S. et CLOUGH G.W. (1968), Behavior of granular materials at high stresses, Proceedings A.S.C.E. Journal of Soil Mechanics and Foundations Division, Vol. 94, n SM3, May 1968, pp. 661-688. 